\title{
Effects of Amino Acid Insertions in the Cysteine-Rich Domain of the MHV-A59 Spike Protein on Cell Fusion
}

\author{
KEVIN W. CHANG AND JAMES L. GOMBOLD \\ Department of Microbiology and Immunology, Louisiana State University Health Sciences \\ Center, Shreveport, LA
}

\section{INTRODUCTION}

The spike protein is a key determinant in the pathogenesis of mouse hepatitis virus strain A59 (Hingley et al., 1994). The spike is a type I viral envelope glycoprotein that mediates receptor-binding and viral entry through virus-cell fusion. In addition, when present on the cell surface, the spike can also mediate cell-cell fusion between infected and neighboring cells. During maturation, the spike protein is post-translationally cleaved into two $90 \mathrm{kDa}$ subunits, S1 and S2. Unlike other viral fusion proteins, fusion mediated by the spike protein does not require a cleavage step that liberates a fusion peptide on the N-terminus of the membrane bound subunit (Bos et al., 1997; Bos et al., 1995; Gombold et al., 1993). Instead, a portion of heptad repeat 1 has been suggested to serve as an internal fusion peptide (Luo and Weiss, 1998a; Luo and Weiss, 1998b). In the current model of fusion, fusion peptides are proposed to mediate lipid mixing between the outer leaflets of the viral envelope and cell membrane. This leads to an intermediate stage, termed hemifusion, in which the inner leaflets remain intact. Resolution of hemifusion is thought to be mediated by the transmembrane anchor. Among coronaviruses, there is significant sequence conservation within the spike transmembrane anchor and cysteine-rich (cys) domain. Both the transmembrane anchor and the cys domain of the spike protein have been shown to be necessary for cell-cell fusion activity (Bos et al., 1995; Chang et al., 2000). In this report, we examine how changes in the length of the

The Nidoviruses (Coronaviruses and Arteriviruses).

Edited hy Fhud Lavi et al.. Kluwer Academic/Plenum Publishers. 2001. 
transmembrane anchor and the consequent change in spacing of the cys domain affect cell fusion activity.

\section{MATERIAL AND METHODS}

\subsection{Cells and virus}

DBT cells were grown in Dulbecco's Modified Eagles Medium (DMEM) containing 10\% fetal bovine serum. Mouse hepatitis virus strain A59 was propagated and titrated by plaque assay on DBT cells. Vaccinia virus WR and the T7 RNA polymerase recombinant vTF7.3 (Fuerst et al., 1987; Fuerst et al., 1986) were prepared as described previously (Chang et al., 2000).

\subsection{PCR mutagenesis}

PCR mutagenesis was performed as described previously (Chang et al., 2000) using mutagenic primers to generate the desired mutations. The resulting PCR products were ligated into the cDNA of the wild-type spike protein under transcriptional control of the T7 RNA polymerase promoter.

\section{$2.3 \quad \beta$-galactosidase-fusion assay}

A fusion assay based on the expression of $\beta$-galactosidase, described originally by Nussbaum et al. (1994), was modified for use with the MHV spike protein as previously described (Chang et al., 2000). Briefly, effector DBT cells were infected with VTF7.3, a recombinant vaccinia virus encoding and expressing the T7 RNA polymerase at $10 \mathrm{PFU}$ per cell for $1 \mathrm{hr}$ at $37^{\circ} \mathrm{C}$. The cells were then washed once with PBS and transfected with plasmid DNA encoding the spike protein under the control of the T7 RNA polymerase promoter and EMC IRES sequence. Reporter cells were infected with wild-type vaccinia virus, strain WR, for $1 \mathrm{~h}$ at $37^{\circ} \mathrm{C}$. This was followed by transfection with plasmid DNA encoding lac $Z$ under the control of the T7 RNA polymerase promoter and EMC IRES sequence. At $4 \mathrm{~h}$ posttransfection the effector and reporter cells were removed with trypsin, mixed, and incubated for $4 \mathrm{hr}$ at $37^{\circ} \mathrm{C}$ in a 96-well plate. Following the incubation, the cells were lysed by adding Triton X-100 in PBS to a final concentration of $1 \%$. The level of $\beta$-galactosidase expression was measured by mixing aliquots of the cell lysate with the $\beta$-galactosidase substrate, CPRG (Boehringer Mannheim), and measuring the optical density at 570 $\mathrm{nm}$. 


\section{$2.4 \quad$ Immunofluorescence}

Cell surface expression of the wild type and mutant spike proteins was examined by immunofluorescence staining. At six hours post-transfection, the cells were incubated with polyclonal goat anti-spike serum AO4 (kindly provided by Dr. Kathryn Holmes, University of Colorado Health Sciences Center), washed, and then fixed in $4 \%$ paraformaldehyde in phosphate buffered saline. The cells were then incubated in fluorescein-conjugated rabbit anti-goat IgG, washed, and examined for fluorescence on an Olympus BX-50 fluorescence microscope.

\section{RESULTS AND DISCUSSION}

\subsection{Extension of the spike transmembrane anchor}

Chimeric spike proteins that substitute the A59 transmembrane anchor with the anchor from glycoprotein $\mathrm{D}(\mathrm{gD})$ of herpes simplex virus type 1 are fusion defective (Chang et al., 2000). The cys domain of the spike, located immediately downstream of the membrane spanning domain, is also required for membrane fusion, but it cannot restore fusion activity to fusion defective A59:gD chimeric spike proteins. The region of the A59 transmembrane anchor upstream of the cys domain is four amino acids shorter than the $\mathrm{gD}$ anchor (Fig. 1A). To determine if the extension of the transmembrane anchor contributed to the fusion negative phenotypes of the chimeric spike proteins, PCR mutagenesis was used to generate insertion mutants of the wild type A59 spike protein that contained either one, two, or all four of the additional amino acids present at the C-terminus of $\mathrm{gD}$ anchor (Fig. 1A). The insertion of just one amino acid (valine, Ins-V) immediately upstream of the cys domain reduced the fusion activity by $98 \%$ while the insertion of two or four amino acids (Ins-VY or Ins-VYWM, respectively) reduced fusion activity by $99 \%$ or greater (Fig. 1B). Membrane fusion requires that the spike protein be transported to the cell surface. Consequently, expression of wild type or mutant spike proteins on the cell surface was examined by immunofluorescence using an anti-spike serum (Fig. 2). Each of the mutant spike proteins was present on the cell surface in levels comparable to wild type spike, indicating that the insertion of either one, two, or four amino acids between the hydrophobic membrane-spanning domain and the cys domain does not interfere with transport of the protein to the cell surface. The increased size of the transmembrane anchor that results from these mutations may affect fusion by altering the position of the cysteine-rich domain relative to the transmembrane anchor. 
(A)

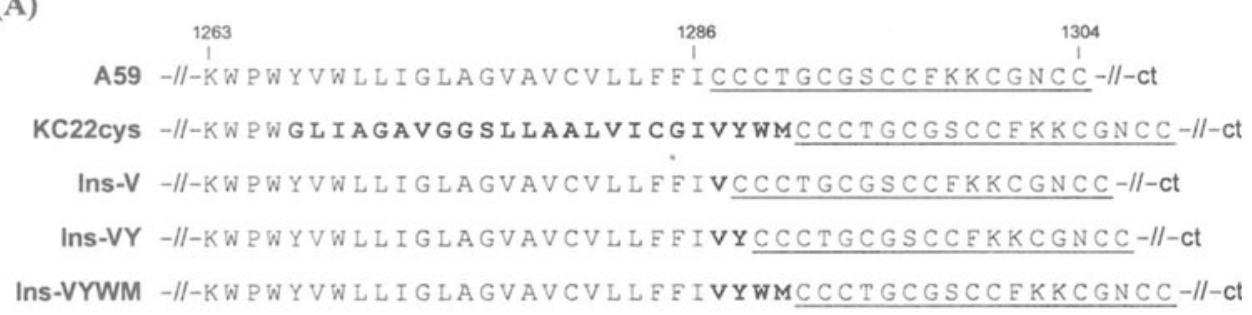

(B)

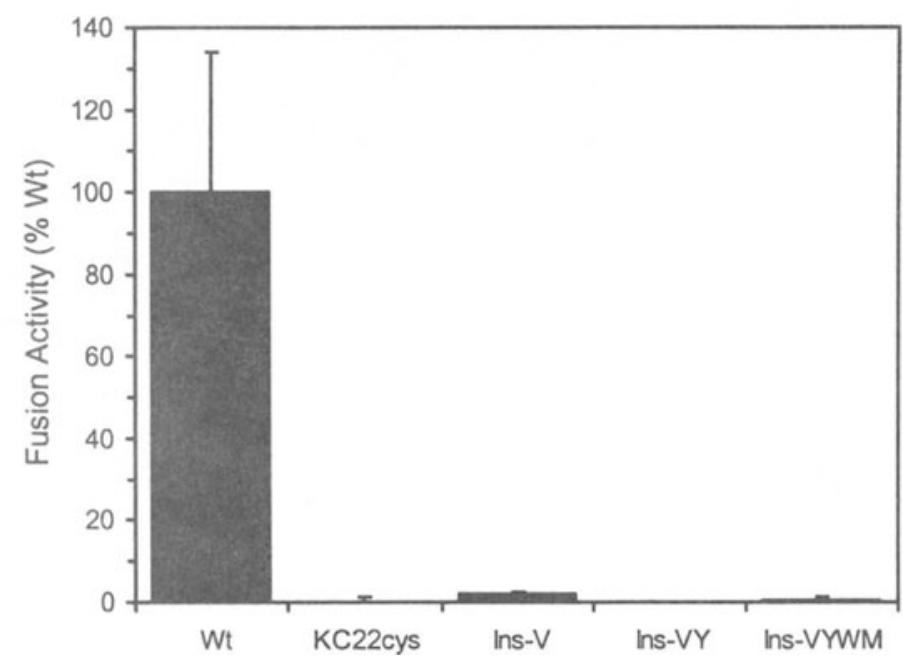

Figure 1. Amino acid insertions upstream of the spike cys domain prevents cell-cell fusion. (A) Sequence of the hydrophobic region of the membrane-spanning domain (amino acids 1263 to 1286 ) and cysteine-rich domain (underlined, amino acids 1287 to 1304) of the wild type A59 and chimeric spike proteins. KC22cys is chimeric spike protein possessing the majority of the HSV gD transmembrane anchor (bold face) fused to the A59 cys domain and cytoplasmic tail (ct) and is fusion defective as shown previously (Chang et al., 2000). Three mutants were created using PCR mutagenesis techniques to insert one (Ins-V), two (Ins-VY) or all four (Ins-VYWM) of the amino acids present at the C-terminus of the $\mathrm{gD}$ transmembrane anchor. (B) Fusion activities of the wild type $(\mathrm{Wt})$ and mutant spike proteins were measured as described in Materials and Methods. The data were normalized to wild type and plotted as the mean percent fusion \pm standard deviation.

\subsection{Single alanine insertions within the cysteine-rich domain}

The insertion of a single valine residue immediately upstream of the cys domain interfered strongly with fusion, but it was unclear if this resulted from the position of the insertion or the nature of the amino acid that was 
inserted. To address this question, single alanine residues, which have a smaller side group and are less hydrophobic than valine, were inserted immediately upstream of the cys domain or at various positions within the domain. When a single alanine was inserted immediately upstream of the cys domain at position 1287, fusion activity decreased $75 \%$ compared to wild type spike (Fig. 3). Even though the reduction in fusion was not as large as that observed with the valine insertion, this result implies that the position of the insertion is a critical factor contributing to the inhibition of fusion.

To further examine this issue, we constructed a series of mutants in which single alanine residues were inserted at several sites downstream of position 1287 but still within the cys domain. Measurement of cell-cell fusion activity for each of the mutants showed that insertion of alanine at position 1290 (A1290) reduced fusion nearly 80\% (Fig. 3). In contrast, insertions at positions 1294,1298 , or 1303 did not negatively affect cell-cell fusion activity of the spike. Thus, fusion was affected when insertions were placed at positions up to and including amino acid 1290. Since we did not examine mutants with insertions between positions 1290 and 1294, we do not know if the region sensitive to insertion mutations extends farther downstream. These results suggest that the position of one or more amino acids in the cys domain upstream of and including threonine 1290 (and possible glycine 1293) may be crucial for fusion to occur properly. It is interesting to note that two glycine residues at positions 1291 and 1293 are found in the cysteine-rich domain of A59 and that both of these residues are conserved for the antigenic group II coronaviruses (Chang et al., 2000). In addition, glycine 1291 is conserved among all coronavirus spike proteins that we have examined. It will be necessary to examine additional mutants to determine if the position of either or both of these two glycines is important for fusion.

Glycines that lie within the transmembrane domain of viral fusion proteins have been suggested to function as "glycine hinges" (Cleverley and Lenard, 1998). These hinges are proposed to allow for flexibility within the transmembrane anchor and thereby promote disruption of the hemifusion diaphragm, an intermediate in the fusion process. If the glycines that are conserved in the A59 spike cys domain are functionally important during fusion, we would anticipate that insertion of alanines upstream of either or both would interfere with fusion similar to the alanine insertions at positions 1287 and 1290. In addition, mutation of glycine 1291 and/or glycine 1293 would be predicted to reduce cell-cell fusion activity of the spike. This would be consistent with our previous observation that deletion of the cysteine-rich domain, where both glycines reside, abrogates fusion activity but does not prevent hemifusion (Chang et al., 2000). 

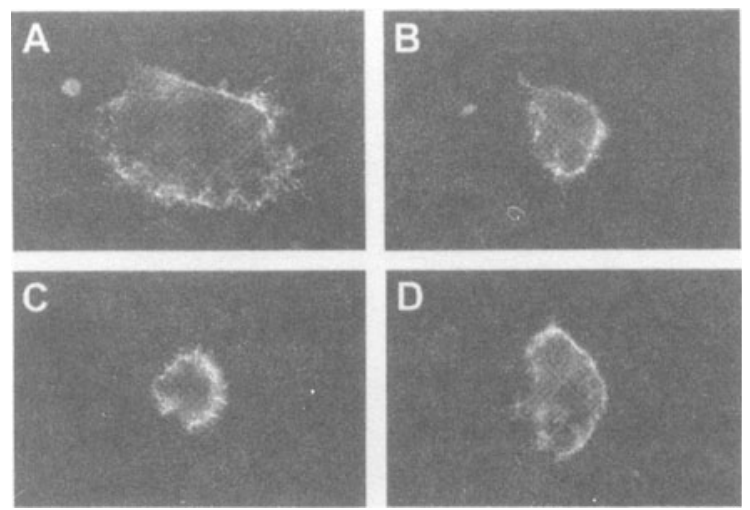

Figure 2. Mutant spike proteins are expressed on the cell surface. Mutant spike proteins were expressed in DBT cells and the cells were stained by immunofluorescence as described in Materials and Methods. (A) Wild type spike, (B) Ins-V, (C) Ins-VY, (D) Ins-VYWM.

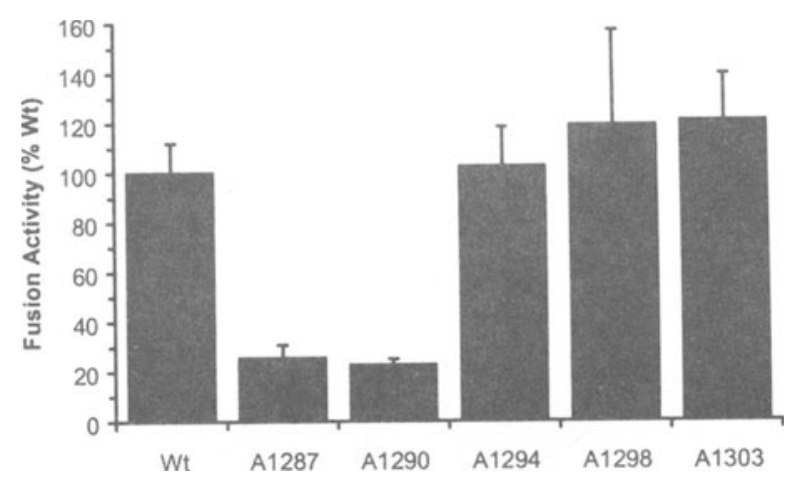

Figure 3. Alanine insertions upstream of position 1290 inhibit cell-cell fusion. PCR mutagenesis was used to generate a series of spike mutants in which a single alanine residue was inserted upstream of the cys domain (position 1287, A1287) or at various positions within the domain (A1290 to A1303). Fusion activities of the wild type (Wt) and mutant spike proteins were measured as described in Materials and Methods. The data were normalized to wild type and plotted as the mean percent fusion \pm standard deviation.

\section{ACKNOWLEDGEMENTS}

We thank Dr. Kay Holmes for providing the $\mathrm{AO} 4$ antiserum and David Ramage and Laura Perkins for the excellent technical assistance. This work was supported by grant LEQSF-RD-A-17 from the Louisiana Education Quality Support Fund and by the Centers for Excellence in Cancer Research and in Arthritis and Rheumatology at the Louisiana State University Health Sciences Center in Shreveport. 


\section{REFERENCES}

Bos, E. C., Luytjes, W., and Spaan, W. J. M. (1997). The function of the spike protein of mouse hepatitis virus strain a59 can be studied on virus-like particles: cleavage is not required for infectivity. J. Virol. 71(12), 9427-9433.

Bos, E. C. W., Heijnen, L., Luytjes, W., and Spaan, W. J. M. (1995). Mutational analysis of the murine coronavirus spike protein: effect on cell-to-cell fusion. Virology 214, 453-463.

Chang, K. W., Sheng, Y., and Gombold, J. L. (2000). Coronavirus-induced membrane fusion requires the cysteine-rich domain in the spike protein. Virology 269, 212-224.

Cleverley, D. Z., and Lenard, J. (1998). The transmembrane domain in viral fusion: Essential role for a conserved glycine residue in vesicular stomatitis virus $\mathrm{G}$ protein. Proc. Natl. Acad. Sci. USA 95, 3425-3430.

Fuerst, T. R., Earl, P. L., and Moss, B. (1987). Use of a hybrid vaccinia virus-T7 RNA polymerase system for expression of target genes. Mol. Cell Biol. 7(7), 2538-2544.

Fuerst, T. R., Niles, E. G., Studier, F. W., and Moss, B. (1986). Eukaryotic transientexpression system based on recombinant vaccinia virus that synthesizes bacteriophage $\mathrm{T} 7$ RNA polymerase. Proc. Natl. Acad. Sci. 83, 8122-8126.

Gombold, J. L., Hingley, S. T., and Weiss, S. R. (1993). Fusion-defective mutants of mouse hepatitis virus A59 contain a mutation in the spike protein cleavage signal. J. Virol. 67, 4504-4512.

Hingley, S. T., Gombold, J. L., Lavi, E., and Weiss, S. R. (1994). MHV-A59 fusion mutants are attenuated and display altered hepatotropism. Virology 200, 1-10.

Luo, Z., and Weiss, S. R. (1998a). Roles in cell-to-cell fusion of two conserved hydrophobic regions in the murine coronavirus spike protein. Virology 244, 483-494.

Luo, Z. L., and Weiss, S. R. (1998b). Mutational analysis of fusion peptide-like regions in the mouse hepatitis virus strain A59 spike protein. In Coronaviruses and Arteriviruses (L. Enjuanes, S.G. Siddel and W Spann, eds) Plenum Press, New York, pp17-23.

Nussbaum, O., Broder, C. C., and Berger, E. A. (1994). Fusogenic mechanisms of envelopedvirus glycoproteins analyzed by a novel recombinant vaccinia virus-based assay quantitating cell fusion-dependent reporter gene activation. J.Virol. 68, 5411-5422. 